\title{
STABILITY AND HOPF BIFURCATION ANALYSIS OF A MATHEMATICAL MODEL IN TUMOR ANGIOGENESIS
}

\author{
Serdal PAMUK ${ }^{1,}$, İrem ÇAY ${ }^{1}$ \\ ${ }^{\mathbf{1}}$ Department of Mathematics, Faculty of Science and Arts, University of Kocaeli, Kocaeli, Turkey
}

\begin{abstract}
This work has been presented at the "International Conference on Mathematics and Engineering, 10-12 May, 2017, Istanbul, Turkey". In this paper we introduce a stability and Hopf bifurcation analysis of a reaction diffusion system which models the interaction between endothelial cells and the inhibitor. Then, we investigate the stability of the positive equilibrium solutions under some conditions. We also show the existence of a Hopf bifurcation and provide some figures to show that the equilibrium solutions are indeed asymptotically stable.
\end{abstract}

Keywords: Equilibrium solution, Hopf bifurcation, Asymptotically stable

\section{INTRODUCTION}

Angiogenesis is the physiological process through which new blood vessels form from pre-existing vessels. It is a normal and vital process in growth and development, as well as in wound healing and in the formation of granulation tissue. However, it is also a fundamental step in the transition of tumors from a benign state to a malignant one, leading to the use of angiogenesis inhibitors in the treatment of cancer. The essential role of angiogenesis in tumor growth was first proposed in 1971 by Judah Folkman, who described tumors as "hot and bloody". The whole mathematical model is studied in [8, 9]. Here we just study the submodel of our model which consists of endothelial cell and angiostatin (as inhibitor) equations only.

Therefore we consider the following initial - boundary value problem:

$$
\begin{aligned}
& \frac{\partial N}{\partial t}=D_{N} \nabla^{2} N+Q(\kappa)\left\{N\left[\theta(1-N)+G\left(C_{A}\right) \frac{\partial C_{A}}{\partial t}\right] H\left(C_{A}-C_{A, 0}\right)-\mu N\right\}, \\
& \frac{\partial A}{\partial t}=D_{A} \nabla^{2} A-\frac{\lambda A N}{1+v A}+a_{0} H\left(F_{\min }-F\right),(x, y) \in \Omega, t>0, \\
& \frac{\partial N}{\partial n}=\frac{\partial A}{\partial n}=0,(x, y) \in \partial \Omega, \\
& N(x, y, 0)=N_{0}(x, y), \quad A(x, y, 0)=A_{0}(x, y), \quad(x, y) \in \Omega
\end{aligned}
$$

where $\Omega=\left(0, l_{1}\right) \times\left(0, l_{2}\right), N=N(x, y, t)$ is the endothelial cell density, $A=A(x, y, t)$ is the angiostatin density, and $C_{A}=C_{A}(x, y, t)$ is the active enzyme density, $G$ is the growth fuction, $H$ is the Heavyside fuction, $n$ is the outer normal to $\partial \Omega, D_{N}$ and $D_{A}$ are diffusion coefficients of endothelial cell and angiostatin, respectively, $Q=Q(\kappa)$ is a function of the curvature $\kappa$ such that $Q(0)=0, Q^{\prime}(x) \geq$ 0 . Also, $C_{A, 0}$ and $F_{\min }$ are some threshold values for active enzyme, $C_{A}$, and fibronectin, $F$, respectively, $[8,9], \theta, \mu, \lambda, v$, and $a_{0}$ are some positive constants, $l_{1}$ and $l_{2}$ are the capillary-tumor distance and the length of the capillary, respectively, and $T$ is some reference time for tumor progression. 
If we set

$$
\begin{aligned}
& x^{*}=\frac{x}{l_{1}}, y^{*}=\frac{y}{l_{2}}, t^{*}=\frac{t}{T}, D_{N}^{*}=\frac{D_{N} T}{l_{1}^{2}}, D_{A}^{*}=\frac{D_{A} T}{l_{1}^{2}}, N^{*}=\frac{N}{N_{0}}, A^{*}=\frac{A}{A_{0}}, \lambda^{*}=\lambda T N_{0}, v^{*}=v A_{0}, \\
& C_{A}^{*}=\frac{C_{A}}{C_{A, 0}}, F^{*}=\frac{F}{F_{\min }}
\end{aligned}
$$

the dimensionless system becomes, on dropping the asterisks for algebraic convenience,

$$
\begin{aligned}
& \frac{\partial N}{\partial t}=D_{N} \nabla^{2} N+Q(\kappa)\left\{N\left[\theta(1-N)+G\left(C_{A}\right) \frac{\partial C_{A}}{\partial t}\right] H\left(C_{A}-1\right)-\mu N\right\}, \\
& \frac{\partial A}{\partial t}=D_{A} \nabla^{2} A-\frac{\lambda A N}{1+v A}+a_{0} H(1-F),(x, y) \in \Omega, t>0, \\
& \frac{\partial N}{\partial n}=\frac{\partial A}{\partial n}=0,(x, y) \in \partial \Omega, \\
& N(x, y, 0)=N_{0}(x, y), \quad A(x, y, 0)=A_{0}(x, y), \quad(x, y) \in \Omega .
\end{aligned}
$$

In [1] the authors analyze the stability of the positive equilibrium, Turing instability, and the existence of Hopf and steady-state bifurcations for a predator-prey model with homogeneous Neumann boundary conditions. In [2] the Gierer-Meinhardt model without the saturating term is considered. By the linear stability analysis, the authors not only give out the conditions ensuring the stability and Turing instability of the positive equilibrium but also find the parameter values where possible. They observe that Turing-Hopf and spatial resonance bifurcation can occur. Similarly, in $[4,6]$ the authors consider the Turing-Hopf bifurcation arising from the reaction-diffusion equations, and in [3,5] the authors derive a necessary and sufficient condition for Turing instabilities to occur in two-component systems of reaction-diffusion equations with Neumann boundary conditions. In [7] the authors study the stability analysis of the steady-state solution of a mathematical model in tumor angiogenesis whereas in [10] Hopf bifurcation of a ratio - dependent predator-prey model involving two discrete maturation time delays is considered.

In the following section we obtain the existence of the positive equilibrium, the stability and Hopf bifurcation of the system (1.5)-(1.8).

\section{STABILITY AND HOPF BIFURCATION}

We take $Q(\kappa) \equiv 1, C_{A}>1, F<1, G\left(C_{A}\right) \frac{\partial C_{A}}{\partial t}=\frac{1}{A}$ for biological convenience, thus the system (1.5)(1.8) becomes as follows:

$$
\begin{aligned}
& \frac{\partial N}{\partial t}=D_{N} \nabla^{2} N+N\left[\theta(1-N)+\frac{1}{A}\right]-\mu N, \quad(x, y) \in \Omega, t>0 \\
& \frac{\partial A}{\partial t}=D_{A} \nabla^{2} A-\frac{\lambda A N}{1+v A}+a_{0}, \quad(x, y) \in \Omega, t>0 \\
& \frac{\partial N}{\partial n}=\frac{\partial A}{\partial n}=0,(x, y) \in \partial \Omega, \\
& N(x, y, 0)=N_{0}(x, y), \quad A(x, y, 0)=A_{0}(x, y), \quad(x, y) \in \Omega .
\end{aligned}
$$

We solve the following equations to find the equilibrium solutions of system (2.1)-(2.2): 


$$
\begin{aligned}
& f(N, A)=N \theta(1-N)+\frac{N}{A}-\mu N=0, \\
& g(N, A)=-\frac{\lambda A N}{1+v A}+a_{0}=0,
\end{aligned}
$$

which is

$$
\left(N_{0}, A_{0}\right)=\left(\frac{a_{0}(\theta-\mu-v)}{a_{0} \theta-\lambda}, \frac{a_{0} \theta-\lambda}{\lambda(\theta-\mu)-a_{0} v \theta}\right)
$$

Here we assume $\frac{a_{0}(\theta-\mu-v)}{a_{0} \theta-\lambda}>0, \frac{a_{0} \theta-\lambda}{\lambda(\theta-\mu)-a_{0} v \theta}>0$, for biological purposes.

We obtain the following linear operator $M$ of the system (2.1)-(2.4) at this $\left(N_{0}, M_{0}\right)$

$$
M=\left(\begin{array}{cc}
D_{N}\left(\frac{\partial^{2}}{\partial x^{2}}+\frac{\partial^{2}}{\partial y^{2}}\right)+\frac{\partial f}{\partial N}\left(N_{0}, A_{0}\right) & \frac{\partial f}{\partial A}\left(N_{0}, A_{0}\right) \\
\frac{\partial g}{\partial N}\left(N_{0}, A_{0}\right) & D_{A}\left(\frac{\partial^{2}}{\partial x^{2}}+\frac{\partial^{2}}{\partial y^{2}}\right)+\frac{\partial g}{\partial A}\left(N_{0}, A_{0}\right)
\end{array}\right)
$$

It is well known that the boundary value problem

$\emptyset^{\prime \prime}=\mu \emptyset, \quad(x, y) \in \Omega$,

$\emptyset_{x}(0, y, t)=\emptyset_{y}(x, 0, t)=\emptyset_{x}(1, y, t)=\emptyset_{y}(x, 1, t)=0$,

has eigenvalues $\mu_{n m}=\left(n^{2}+m^{2}\right) \pi^{2},(n, m=1,2, \ldots)$, with corresponding eigenfunc-tions $\emptyset_{n m}=(\cos n \pi x)(\cos m \pi y)$. Thus,

$$
M_{n, m}=\left.M\right|_{\emptyset_{n, m}}=\left(\begin{array}{cc}
-D_{N}\left(n^{2}+m^{2}\right)+f_{N} & f_{A} \\
g_{N} & -D_{A}\left(n^{2}+m^{2}\right)+g_{A}
\end{array}\right),
$$

where

$$
\begin{gathered}
f_{N}=\frac{\partial f}{\partial N}\left(N_{0}, A_{0}\right)=\frac{a_{0} \theta(-\theta+\mu+v)}{a_{0} \theta-\lambda}<0 \\
f_{A}=\frac{\partial f}{\partial A}\left(N_{0}, A_{0}\right)=-\frac{a_{0}(\theta-\mu-v)\left(\lambda(\theta-\mu)-a_{0} v \theta\right)^{2}}{\left(a_{0} \theta-\lambda\right)^{3}}<0,
\end{gathered}
$$

and

$$
\begin{gathered}
g_{N}=\frac{\partial g}{\partial N}\left(N_{0}, A_{0}\right)=-\frac{a_{0} \theta-\lambda}{\theta-\mu-v}<0, \\
g_{A}=\frac{\partial g}{\partial A}\left(N_{0}, A_{0}\right)=-\frac{a_{0}\left(\lambda(\theta-\mu)-a_{0} v \theta\right)^{2}}{\lambda\left(a_{0} \theta-\lambda\right)(\theta-\mu-v)}<0 .
\end{gathered}
$$

The characteristic equation of $M_{n, m}$ is then given by

$$
\lambda^{2}-\lambda T_{n m}+D_{n m}=0, \quad n, m=1,2, \ldots,
$$

where

$$
T_{n m}:=\operatorname{trace}\left(M_{n, m}\right)=-D_{N}\left(n^{2}+m^{2}\right)-D_{A}\left(n^{2}+m^{2}\right)+f_{N}+g_{A}<0
$$


and

$$
D_{n m}:=\operatorname{det}\left(M_{n, m}\right)=D_{N} D_{A}\left(n^{2}+m^{2}\right)^{2}-\left(D_{A} f_{N}+D_{N} g_{A}\right)\left(n^{2}+m^{2}\right)+f_{N} g_{A}-g_{N} f_{A} .
$$

We set $H:=n^{2}+m^{2}$, then $D_{n m}$ can be written as

$$
D_{n m}:=f(H)=D_{N} D_{A} H^{2}-\left(D_{A} f_{N}+D_{N} g_{A}\right) H+f_{N} g_{A}-g_{N} f_{A} .
$$

Then the discriminant of $f(H)=0$ is

$$
\Delta_{H}=\left(D_{A} f_{N}-D_{N} g_{A}\right)^{2}+4 D_{N} D_{A} g_{N} f_{A} .
$$

Lemma 2.1: $f(H)=0$ has two roots $H_{1}$ and $H_{2}$, where

$$
H_{1}=\frac{D_{A} f_{N}+D_{N} f_{A}-\sqrt{\Delta_{H}}}{2 D_{N} D_{A}} \text { and } H_{2}=\frac{D_{A} f_{N}+D_{N} f_{A}+\sqrt{\Delta_{H}}}{2 D_{N} D_{A}} \text {. }
$$

Theorem 2.1: If $\theta>\frac{\lambda}{a_{0}}$, the equilibrium point $\left(N_{0}, A_{0}\right)$ is locally asymptotically stable.

Proof: Since $\theta>\frac{\lambda}{a_{0}}$, we have $D_{n m}>0$, for any $n, m=1,2, \ldots$.

Noting that $f_{N}<0$ and $g_{A}^{-}<0$, we obtain $T_{n m}<0$ for any $n, m=1,2, \ldots$. Thus, all eigenvalues of $M_{n, m}$ have negative real parts. That is, the equilibrium point $\left(N_{0}, A_{0}\right)$ is locally asymptotically stable. This completes the proof.

Theorem 2.2: When $\theta<\frac{\lambda}{a_{0}}$, the equilibrium point $\left(N_{0}, A_{0}\right)$ is unstable, if for all $n, m=1,2, \ldots$ the condition $H_{1}<H<H_{2}$ holds.

Proof: Following from $\theta<\frac{\lambda}{a_{0}}$ we get $f_{N} g_{A}-g_{N} f_{A}<0$ and $D_{n m}<0$, since for any $n, m=1,2, \ldots$ the condition $H_{1}<H<H_{2}$ holds.

Corollary 2.1: By the Hopf bifurcation theorem, there is a Hopf bifurcation of the system (2.1)-(2.4) at $\left(N_{0}, A_{0}\right)$ as $\theta$ passes through the critical value $\frac{\lambda}{a_{0}}$.

\section{NUMERICAL EXAMPLES}

In the following examples we take $N_{0}(x, y)=(\sin (\pi x) \sin (\pi y))^{12}$,

$A_{0}(x, y)=(2-\sin (\pi x) \sin (\pi y))^{4}$ as the initial condition functions for endothelial cells and angiostatin, respectively. Here $0 \leq x, y \leq 1$.

Since in both Figure $1 \mathrm{a}$ ) and Figure $1 \mathrm{~b}$ ) the condition $\theta>\frac{\lambda}{a_{0}}$ holds, the more we introduce inhibitor to the system the more endothelial cells (tumor cells) drops down in the density. This is a biologically expected result which means that the solution obtained at $t=10$ is stable. 

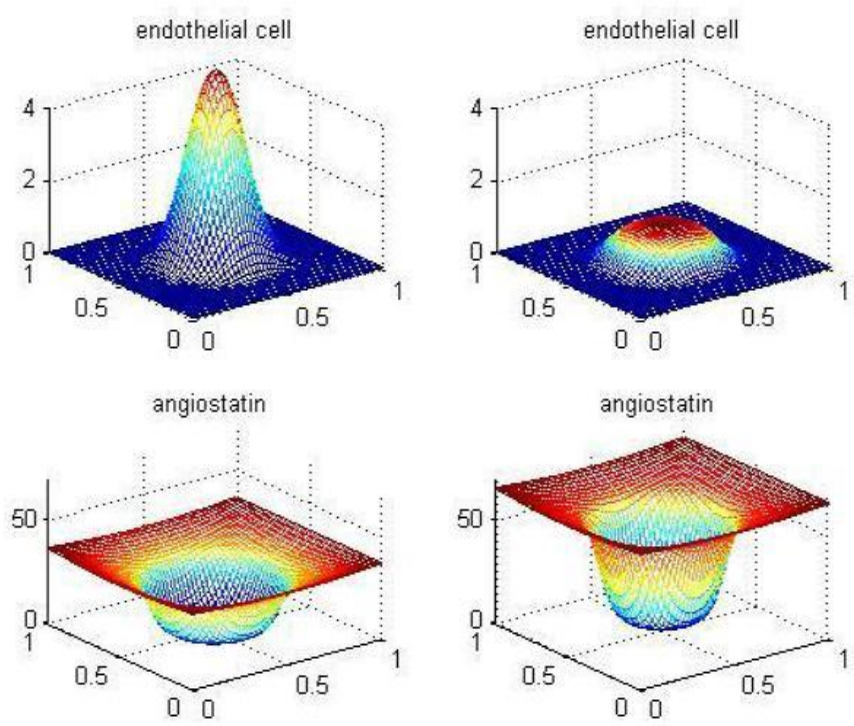

(a)

(b)

Figure 1. $\theta=0.5, \mu=0.2, v=0.014, \lambda=1, t=10, D_{N}=3.6 \times 10^{-6}, D_{A}=6.5 \times 10^{-5}, \theta>\frac{\lambda}{a_{0}}, \quad(a): a_{0}=$ $2.1,(b): a_{0}=5$.

In Figure 2 a), where endothelial cells density drops down, the condition $\theta>\frac{\lambda}{a_{0}}$ holds. However, in Figure 2 b), where endothelial cells density increases, the condition does not hold.
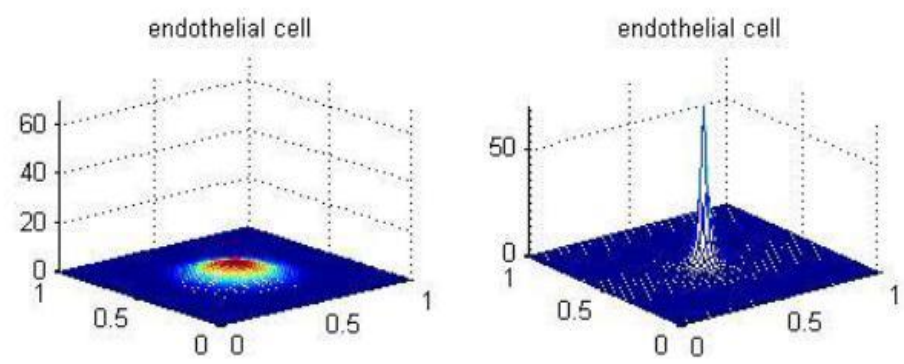

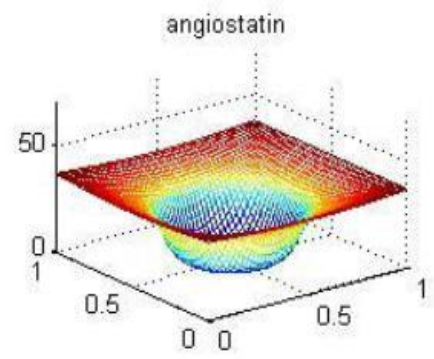

(a)

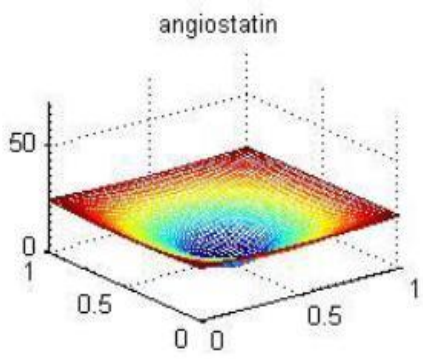

(b)

Figure 2. $a_{0}=2.1, \mu=0.2, v=0.014, \lambda=1, t=10, D_{N}=3.6 \times 10^{-6}, D_{A}=6.5 \times 10^{-5}$, (a): $\theta=0.5$, (b): $\theta=0.2$. 
Figure 3 and Figure 4 show the phase space diagrams of the system (2.5)-(2.6) in the cases $\theta>\frac{\lambda}{a_{0}}$ and $<\frac{\lambda}{a_{0}}$, respectively. In Figure 3 the solution curves tend to the constant steady-state $\left(N_{\mathbf{0}}, A_{\mathbf{0}}\right)=$ $(12,0.18)$, showing that this steady-state is locally asymptotically stable. However, in Figure 4 the solution curves get further away from the constant steady-state $\left(N_{\mathbf{0}}, A_{\mathbf{0}}\right)=(0.303,7.67)$, proving that this steady-state is unstable.

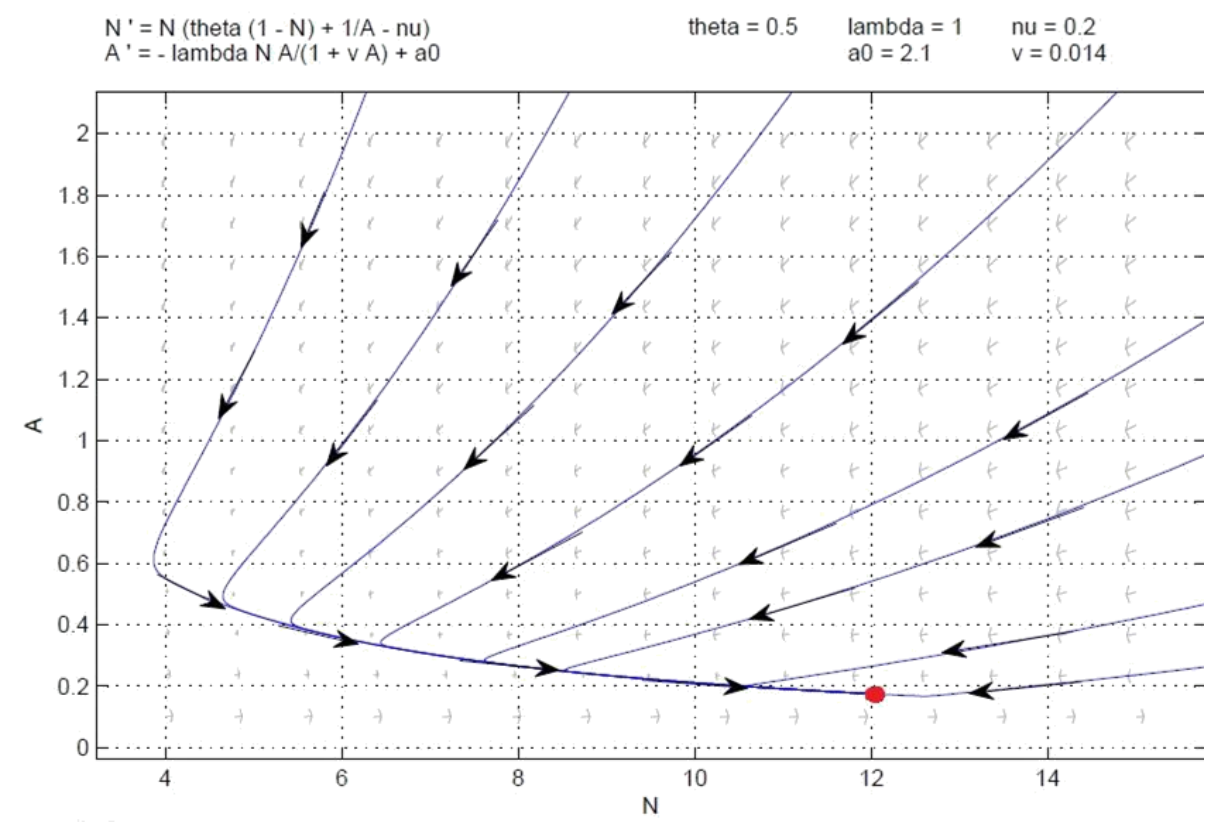

Figure 3. Phase space diagrams of $(N, A)$ with $D_{N}=D_{A}=0, \theta>\frac{\lambda}{a_{0}}$. The solutions tends to the constant steadystate $\left(N_{0}, A_{0}\right)=(12,0.18)$.

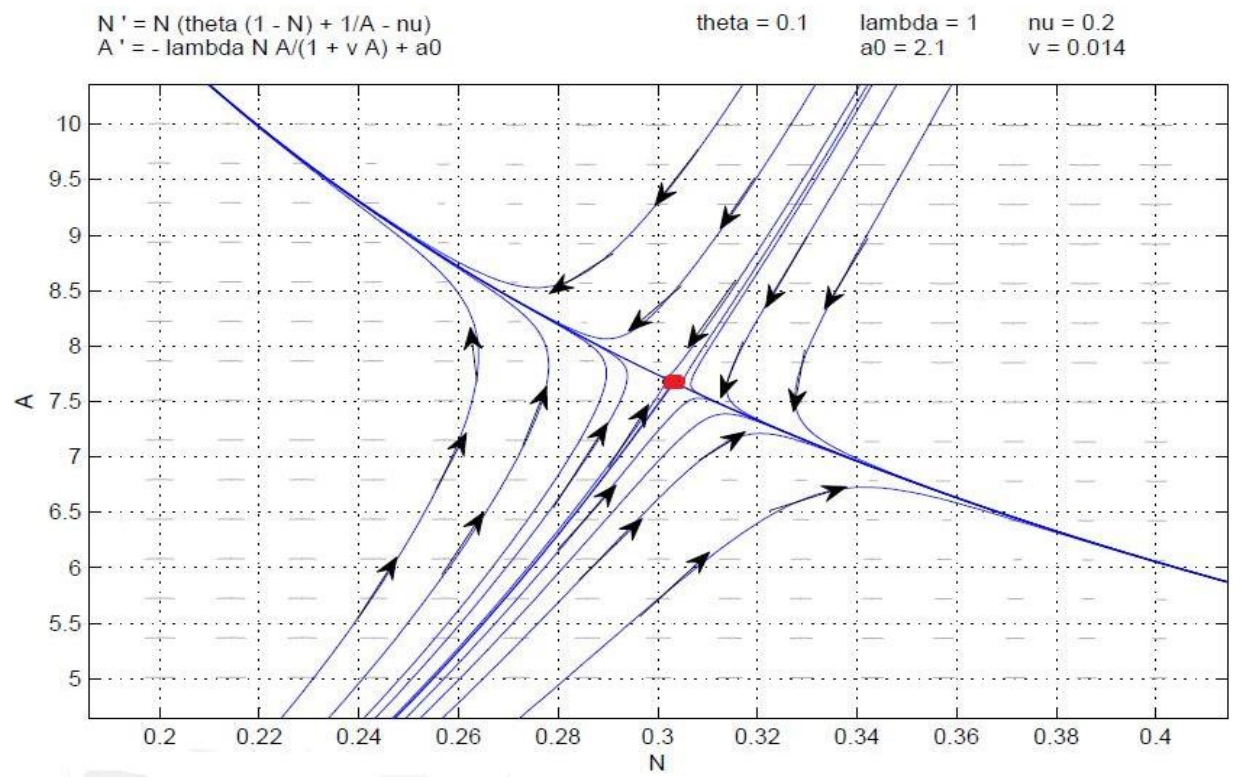

Figure 4. Phase space diagrams of $(N, A)$ with $D_{N}=D_{A}=0, \theta<\frac{\lambda}{a_{0}}$. The solutions get further away from the constant steady-state $\left(N_{0}, A_{0}\right)=(0.303,7.67)$. 
On the other hand, Figure 5 and Figure 6 represents that endothelial cell density, $N(x, y)$ and angiostatin density, $A(x, y)$, respectively, at some increasing time steps. One can easily figure out that in the case $\theta>\frac{\lambda}{a_{0}}$, both $N(x, y)$ and $A(x, y)$ approach to the constant steady-state $\left(N_{\mathbf{0}}, A_{\mathbf{0}}\right)=$ $(12,0.18)$ as time evolves. As a result, we can conclude that there is a Hopf bifurcation of system (2.1)-(2.4) at $\left(N_{\mathbf{0}}, A_{\mathbf{0}}\right)$ as $\theta$ passes through the critical value $\frac{\lambda}{a_{0}}$.

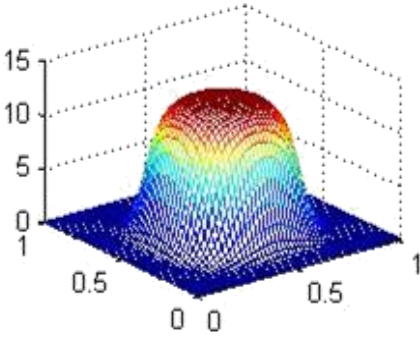

(a)

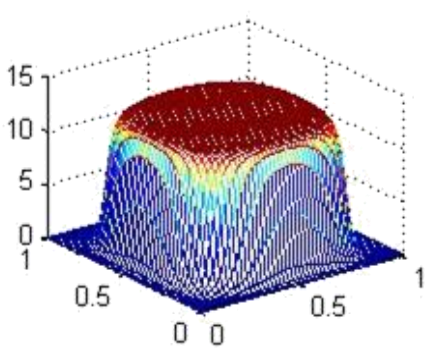

(c)

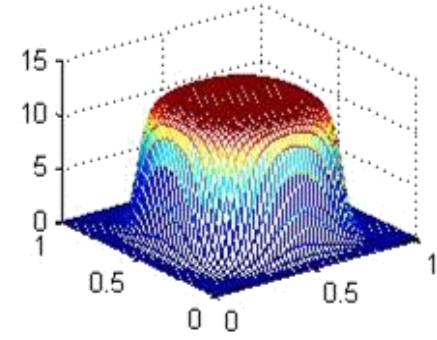

(b)

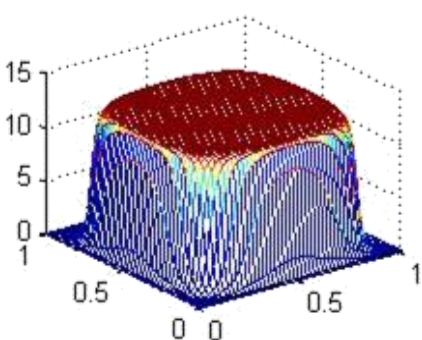

(d)

Figure 5. Graph of $(x, y), D_{N}=D_{A}=0, \theta>\frac{\lambda}{a_{0}}$, the solution tends to the constant steady-state $N=12,(a) t=$ 20,(b) $t=40,(c) t=60,(d) t=80$.

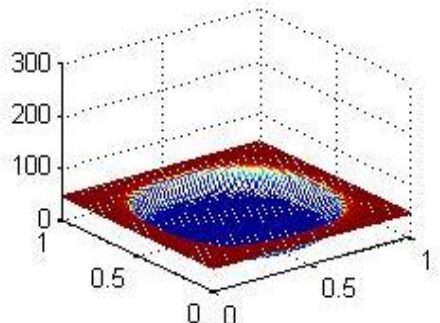

(a)

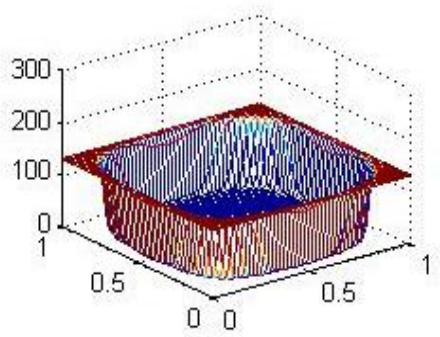

(c)

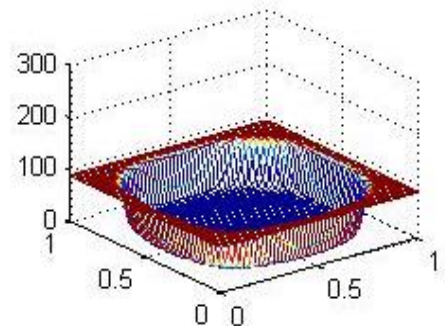

(b)

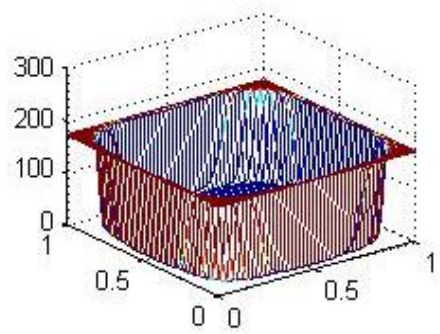

(d)

Figure 6. Graph of $A(x, y), D_{N}=D_{A}=0, \theta>\frac{\lambda}{a_{0}}$, the solution tends to the constant steady-state $\quad A=0.18$, (a) $t=20,(b) t=40,(c) t=60,(d) t=80$. 


\section{CONCLUSION AND RESULTS}

In this paper we have introduced a stability and Hopf bifurcation analysis of a reaction diffusion system which models the interaction between endothelial cells and the inhibitor. We have also investigated the stability of the positive equilibrium solutions under some conditions, and have shown the existence of a Hopf bifurcation. Finally we have provided some figures to show that the equilibrium solutions of the system are indeed asymptotically stable.

\section{ACKNOWLEDGEMENT}

This work has been supported by Scientific and Technological Research Council of Turkey, TUBITAK, project no: 115F538.

\section{REFERENCES}

[1] Tang X, Song Y. Bifurcation analysis and Turing instability in a diffusive predator-prey model with herd behavior and hyperbolic mortality. Chaos, Soli-tons and Fractals 2015; 81: 303-314.

[2] Yang R, Song Y. Spatial resonance and Turing-Hopf bifurcations in the Gierer-Meinhardt model. Nonlinear Analysis: Real World Applications 2016; 31:356-387.

[3] Dil $a$ o R. Turing instabilities and patterns near a Hopf bifurcation. Applied Math-ematics and Computation 2005; 164:391-414.

[4] Ling W, Hongyong Z. Hopf bifurcation and Turing instability of 2-D Lengyel-Epstein system with reaction-diffusion terms. Applied Mathematics and Computation 2013; 219:9229-9244.

[5] Zhang JF, Li WT, Yan XP. Hopf bifurcation and Turing instability in spa-tial homogeneous and inhomogeneous predator-prey models. Applied Mathematics and Computation 2011; 218:18831893.

[6] Song Y, Zhang T, Peng Y. Turing-Hopf bifurcation in the reaction-diffusion equations and its applications. Commun Nonlinear Sci Numer Simulat 2016; 33:229-258.

[7] Pamuk S, Gürbüz A. Stability analysis of the steady-state solution of a mathe-matical model in tumor angiogenesis. AIP Conference Proceedings 2004; 729: 369 .

[8] Levine HA, Pamuk S, Sleeman BD, Hamilton MN. A mathematical model of capillary formation and development in tumour angiogenesis: penetration into the stroma. Bull. Math. Biol 2001; 63:801-863.

[9] Pamuk S, Çay I. A 2D Mathematical Model for Tumor Angiogenesis: The Roles of Endothelials, Pericytes and Macrophages in the ECM. (in preperation).

[10] Karaoglu E, Merdan H. Hopf bifurcation of a ratio - dependent predator-prey model involving two discrete maturation time delays. Chaos, Solitons \& Fractals 2014; 68:159-168. 